\title{
Spontaneous Fluctuations in the Flexible Control of Covert Attention
}

\author{
(D)Anthony W. Sali, ${ }^{1}$ Susan M. Courtney, ${ }^{1,2,3}$ and Steven Yantis ${ }^{1 \dagger}$ \\ ${ }^{1}$ Department of Psychological and Brain Sciences and ${ }^{2}$ Department of Neuroscience, Johns Hopkins University School of Medicine, Baltimore, Maryland \\ 21218, and ${ }^{3}$ F.M. Kirby Research Center for Functional Brain Imaging, Kennedy Krieger Institute, Baltimore, Maryland 21218
}

\begin{abstract}
Spontaneous fluctuations in cognitive flexibility are characterized by moment-to-moment changes in the efficacy of control over attentional shifts. We used fMRI to investigate the neural correlates in humans of spontaneous fluctuations in readiness to covertly shift attention between two peripheral rapid serial visual presentation streams. Target detection response time (RT) after a shift or hold of covert spatial attention served as a behavioral index of fluctuations in attentional flexibility. In particular, the cost associated with shifting attention compared with holding attention varied as a function of pretrial brain activity in key regions of the default mode network (DMN), but not the dorsal attention network. High pretrial activity within the DMN was associated with a greater increase in shift trial RT relative to hold trial RT, revealing that these areas are associated with a state of attentional stability. Conversely, high pretrial activity within bilateral anterior insula and the presupplementary motor area/supplementary motor area was associated with a greater decrease in shift trial RT relative to hold trial RT, reflecting increased flexibility. Our results importantly clarify the roles of the precuneus, medial prefrontal cortex, and lateral parietal cortex, indicating that reduced activity may not simply indicate greater task engagement, but also, specifically, a readiness to update the focus of attention. Investigation of the neural correlates of spontaneous changes in attentional flexibility may contribute to our understanding of disorders of cognitive control as well as healthy variability in the control of spatial attention.
\end{abstract}

Key words: attention; cognitive control; fMRI

Significance Statement

Individuals regularly experience fluctuations in preparatory cognitive control that affect performance in everyday life. For example, individuals are able to more quickly initiate a spatial shift of attention at some moments than at others. The current study revealed that pretrial brain activity in specific cortical regions predicted trial-by-trial changes in participants' abilities to flexibly shift the focus of attention. Intrinsically generated fluctuations in brain activity within several key default mode network regions, as well as within the anterior insula and presupplementary/supplementary motor areas, carried behavioral consequences for preparatory attentional control beyond lapses of attentional engagement. Our results are the first to link intrinsic variation in pretrial brain activity to moment-by-moment changes in preparatory attentional control over spatial selection.

\section{Introduction}

Attentional selection shapes our awareness of the world around us such that physically salient, reward-associated, or goal-relevant stimuli receive preferential representation within the brain and

Received June 15, 2015; revised Nov. 17, 2015; accepted Nov. 24, 2015.

Author contributions: A.W.S. and S.Y. designed research; A.W.S. performed research; A.W.S., S.M.C., and S.Y. analyzed data; A.W.S., S.M.C., and S.Y. wrote the paper.

This research was supported by the National Institutes of Health (Grant R01-DA013165 to S.Y. and S.M.C.) and the National Science Foundation (Grant GRFP DGE-0707427 to A.W.S.). We thank M. Hall for assistance with data collection.

The authors declare no competing financial interests.

†Deceased June 20, 2014.

Correspondence should be addressed to Anthony W. Sali, Center for Cognitive Neuroscience, Duke University, LSRC Box \#90999, Durham, NC 27708. E-mail: anthony.sali@duke.edu.

DOI:10.1523/JNEUROSCI.2323-15.2016

Copyright $\odot 2016$ the authors $\quad 0270-6474 / 16 / 360445-10 \$ 15.00 / 0$ strongly influence our behavior (Desimone and Duncan, 1995; Reynolds et al., 1999; Anderson et al., 2011; Sali et al., 2014). Dysfunctions of attentional control such as lapses of attention, perseveration, and distraction are frequently associated with a variety of clinical syndromes such as attention deficit hyperactivity disorder (ADHD; Barkley et al., 1997), substance abuse (Cools, 2008), and obesity (Volkow et al., 2011). Healthy individuals also regularly experience fluctuations in their abilities to control attention that significantly affect behavior (Bellgrove et al., 2004). However, little is known about the neural basis of these spontaneous fluctuations in preparatory states of attentional control. In the current study, we used fMRI to investigate how dynamic changes in brain activity reflect moment-by-moment fluctuations in individuals' readiness to perform spatial shifts of attention. 
States of preparatory control may range from periods of attentional flexibility, in which individuals are able to rapidly shift attention, to periods of attentional stability, in which shifts of attention are sluggish. Although persistent individual differences and environmental factors likely play a role in the frequency and magnitude of moment-by-moment changes in cognitive flexibility (Sali et al., 2015), intrinsic fluctuations of brain activity may also contribute to these control settings. The relationship between changes in trial-by-trial behavioral performance and ongoing fluctuations in brain activity as measured by fMRI may therefore contribute to our understanding of the neural mechanisms implicated in preparatory cognitive control.

Spontaneous fluctuations in brain activity have been associated with both changes in task engagement (Weissman et al., 2006) and in preparatory cognitive control (Leber et al., 2008; Leber, 2010). Using a task-switching paradigm, Leber and colleagues (2008) examined the relationship between changes in pretrial brain activity and the size of behavioral task switch costs. A pretrial increase of activity within a group of cortical and subcortical regions, including the left superior parietal lobule, anterior cingulate cortex, left inferior parietal lobule, right middle frontal gyrus (MFG), and the left putamen, was associated with an increase in cognitive flexibility. In a similar study, spontaneous fluctuations of pretrial activity within the left MFG predicted the degree to which a salient distractor item captured attention on a trial-by-trial basis (Leber, 2010).

In the current study, we extended these previous investigations of spontaneous changes in preparatory control to the domain of goal-oriented covert spatial attention. It is possible that the frontoparietal neural mechanisms involved in the execution of covert attentional control, referred to here as the dorsal attention network (DAN), will also predict moment-by-moment changes in preparatory attentional flexibility (Yantis et al., 2002; Serences and Yantis, 2006; Chiu and Yantis, 2009; Esterman et al., 2009; see Corbetta and Shulman, 2002 for a review). Conversely, a collection of brain regions comprising the default mode network (DMN) have also been linked to moment-by-moment changes in attentional states. These brain regions, including the precuneus, ventromedial prefrontal cortex (vmPFC), and lateral parietal cortex, show correlated activity among themselves at rest in the absence of an overt task and are negatively correlated with frontal and parietal cognitive control regions (Raichle et al., 2001). Therefore, an alternative possibility is that attentional flexibility is associated with activity changes within these regions that have previously been associated with attentional disengagement. Importantly, such a finding would extend the role of DMN regions beyond mere task engagement and would suggest that spontaneous fluctuations in brain activity carry differential consequences for behavioral performance depending on whether a shift of attention is necessary.

\section{Materials and Methods}

Participants. Twenty adults (11 women, 1 participant did not report gender) ranging in age from 18 to 32 years $(\mathrm{M}=21.8, \mathrm{SD}=3.37)$ completed a $2 \mathrm{~h}$ fMRI scanning session in exchange for monetary compensation. Eighteen of the participants were selected based on task performance during an earlier behavioral screening session. These participants completed at least 10 runs of the exact task used in the scanner before participation in the fMRI study. The remaining two participants were both highly experienced in performing similar experimental tasks and therefore completed no additional training for the current study. All participants had normal or corrected-to-normal vision and all but one was right handed. The protocol was approved by the Institutional

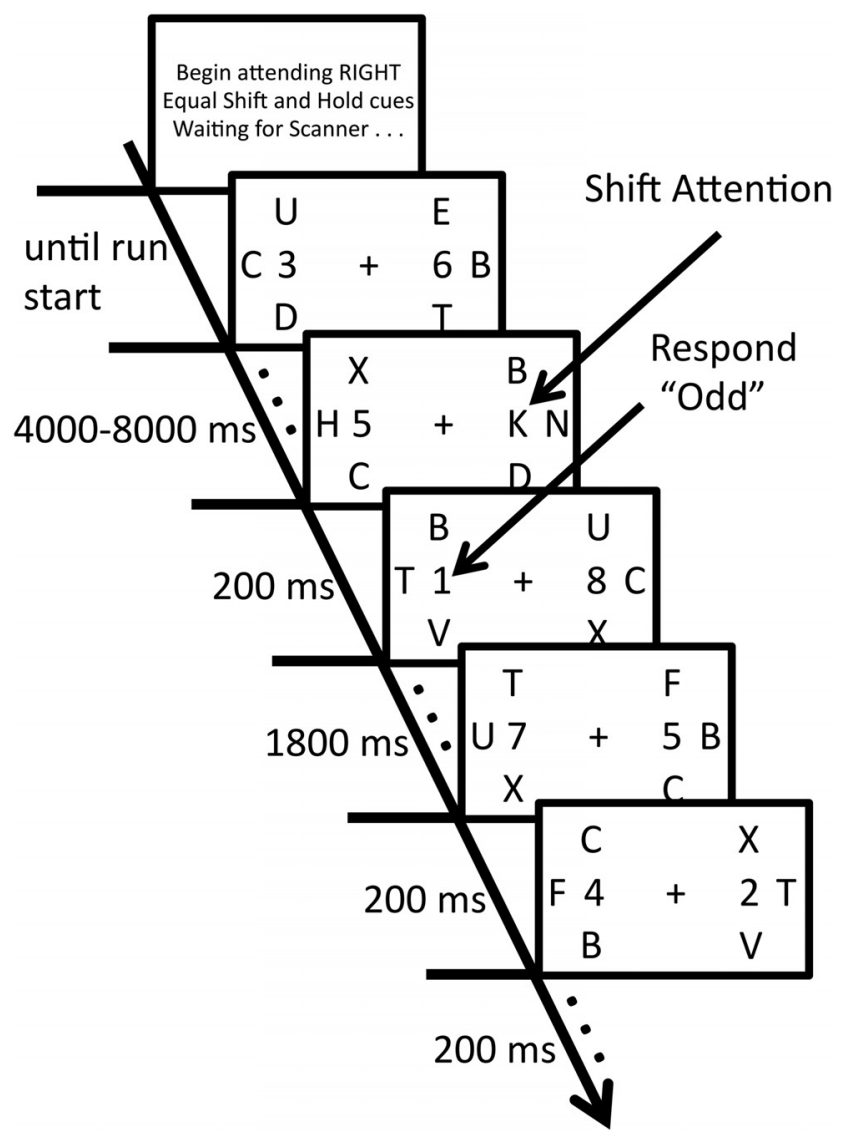

Figure 1. Experimental task. Participants monitored one of two peripheral task-relevant RSVP streams for the appearance of a visual cue. Letter cues instructed participants to shift or hold attention. Participants made a speeded parity judgment for stimuli appearing in the cued location immediately after each cue presentation.

Review Boards of the Johns Hopkins University and the Johns Hopkins Medical Institutions and all participants gave written, informed consent.

Stimuli and procedure. All stimuli were displayed on a back-projection screen located in the bore of the scanner, which participants viewed through a mirror attached to the head coil. The stimuli consisted of multiple white rapid serial visual presentation (RSVP) streams of alphanumeric characters, each displayed for $200 \mathrm{~ms}$ with no gap appearing against a black background (Fig. 1). At any given moment, the participant attended either to the stream positioned to the left or the one positioned to the right of a central fixation point $\left(0.5^{\circ}\right.$ by $0.5^{\circ} ; 3.00^{\circ}$ of visual angle from the fixation point to the center of each target stream). Each alphanumeric character subtended $\sim 0.56^{\circ}$ by $1.12^{\circ}$ of visual angle. Stimulus presentation was controlled by the Psychophysics Toolbox (version 3.08; Brainard, 1997) running in MATLAB.

Each run began with the presentation of the words either "Begin attending RIGHT" or "Begin attending LEFT" for $\sim 8 \mathrm{~s}$ that indicated the first to-be-attended RSVP stream. Participants monitored this stream for the appearance of an embedded visual cue to either shift attention to the opposite stream or to hold attention at that location. For the majority of each run, these 2 task-relevant streams contained randomly generated digits ranging from 1 to 8 . Occasionally, the letter " $A$ " or the letter " $K$ " appeared in the attended stream, cuing participants to either continue to hold their attention at the currently attended location or to shift attention to the other stream. For half of the participants, the letter " $K$ " cued a covert shift of attention to the opposite RSVP stream and the letter " $A$ " cued participants to hold attention at the location of the cue. The remainder of the participants completed the task with the opposite cue mapping. Participants received 18 shift and 18 hold cues during each run. There were 12 runs during the scanning session, for a total of 216 cue events of each type. All cues were presented in a random order with the only 
constraint being that no more than 3 cues of the same type (shift or hold) could be presented consecutively. Three flanking streams surrounding each of the target streams $\left(2.16^{\circ}\right.$ center-to-center $)$ consisted of taskirrelevant randomly generated letters (excluding $\mathrm{A}, \mathrm{K}, \mathrm{I}, \mathrm{O}$, and $\mathrm{W}$ ).

Immediately after the offset of the letter cue, participants made a speeded judgment regarding the parity of digits appearing at the cued location. During a $2 \mathrm{~s}$ response period, all digits appearing in the cued stream were of the same parity (either all even or all odd). Participants received instructions before beginning the task that they were to respond based on the first digit identified during this response window while still maintaining a high level of accuracy. Because all of the target digits had the same parity, behavioral response time (RT) served as an indicator of trial-by-trial attentional fluctuations. Specifically, the amount of slowing in RT for a shift trial compared with a hold trial indicated the subject's current state of attentional flexibility. Digits appearing in the noncued stream remained randomly generated during the response window. Participants made all responses with two MR-compatible buttons. Participants pressed one button with their left thumb and the other with their right thumb and we counterbalanced the response mapping of parity across participants. After the $2 \mathrm{~s}$ response window, the digits in both target streams were again randomly generated. Any presses made after the $2 \mathrm{~s}$ response window were scored as incorrect. After making a response, participants maintained attention at the peripheral stream that previously contained the target digits until the appearance of the next letter cue. To discourage uncued shifts of attention, participants received instructions that, after a response, the next cue would appear in the last attended location. For example, if participants were cued to attend to the left stream on trial $n$, the attention cue ("K" or "A") would appear in the left stream during trial $n+1$. The next cue appeared 4,6 , or $8 \mathrm{~s}$ after the previous cue presentation.

In addition to the functional data obtained during task performance, we acquired a high-resolution anatomical scan for registration of the functional images to a standardized template as well as a resting state scan to independently define the DMN and DAN regions of interest (ROIs). Participants fixated a central cross with no explicit task for the duration of the $5 \mathrm{~min}$ resting-state scan. The remainder of the scanning session consisted of eight runs of a shortened version of the task in which the frequency of shift and hold cues varied across runs (data not reported here).

fMRI data acquisition and analysis. MR images were acquired with a Phillips Intera 3T scanner at the Kirby Center for Functional Brain Imaging at the Kennedy Krieger Institute in Baltimore, Maryland. For each subject, a high-resolution anatomical scan was acquired with an MPRAGE T1-weighted sequence with an isotropic voxel size of $1 \mathrm{~mm}$ repetition time $(\mathrm{TR})=8.1 \mathrm{~ms}$; echo time $(\mathrm{TE})=3.7 \mathrm{~ms}$, flip angle $=8^{\circ}$, 150 axial slices, $0 \mathrm{~mm}$ gap, SENSE factor $=2$ ]. T2* -weighted echoplanar images were acquired with a 32-channel SENSE head coil in 36 transverse, sequential slices $\left(\mathrm{TR}=2000 \mathrm{~ms}\right.$; $\mathrm{TE}=30 \mathrm{~ms}$; flip angle $=70^{\circ}$; acquisition matrix $=76 \times 76$, field of view $=192.00 \mathrm{~mm} \times 171.79$ $\mathrm{mm} \times 107.50 \mathrm{~mm}$; $0.5 \mathrm{~mm}$ gap; SENSE factor $=2$ ), yielding voxels that were $2.5 \mathrm{~mm}$ isotropic and covering most of the brain. Each EPI scan began with four dummy pulses before the RSVP onset to allow magnetization to reach steady state. We acquired 112 volumes for each of the 12 RSVP runs such that each lasted $\sim 3.7 \mathrm{~min}$. The resting-state scan was identical to the functional runs, with the exception that 150 volumes were acquired for a total of $5 \mathrm{~min}$.

Preprocessing of the data was performed using Analysis of Functional NeuroImages (AFNI; Cox, 1996) with the exception of removing the skull from the anatomical scan to improve image coregistration, which was performed using fsl_anat (Jenkinson et al., 2012). We applied AFNI's nonlinear warping (3dQWarp) to morph each subject's anatomical scan into Talairach stereotaxic space according to the Colin 27 template. All functional images were first corrected for slice time acquisition. Next, we corrected for subject motion and registered each image to the corresponding normalized anatomical scan by applying the parameters from the nonlinear warping. The functional runs were resampled to an isotropic resolution of $2 \mathrm{~mm}$ during coregistration. Last, we performed spatial smoothing with a kernel of $4 \mathrm{~mm}$ full width half maximum and normal-
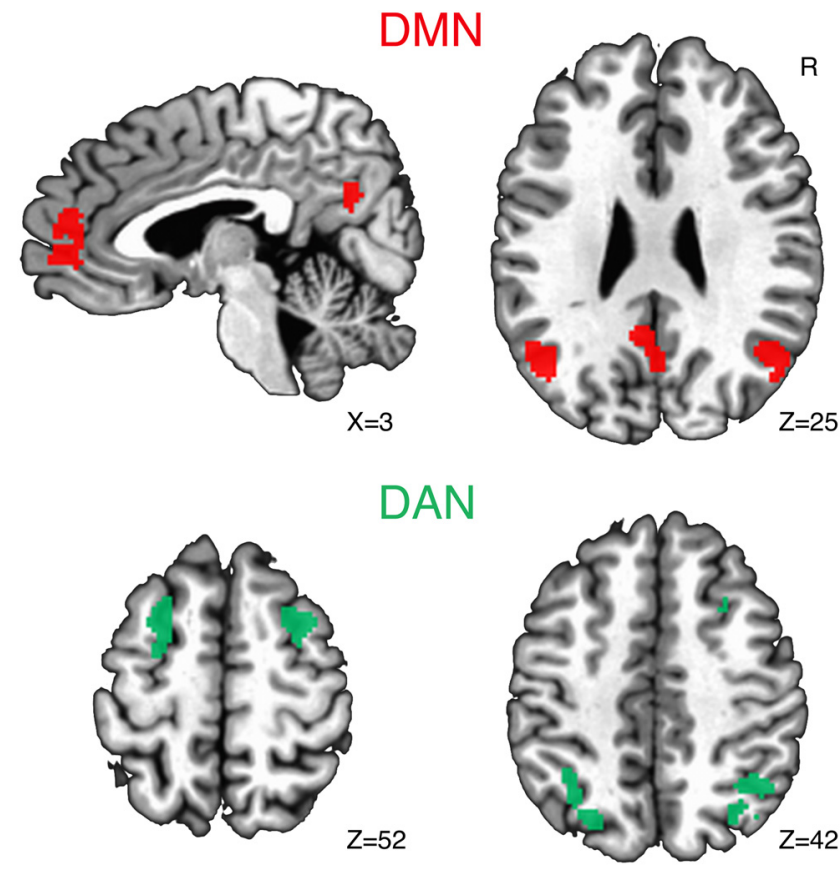

Figure 2. ROls. We independently defined ROls in the DMN and DAN using independent components analysis in the resting-state data. DMN regions consisted of the precuneus, ventromedial PFC, and bilateral lateral parietal cortices. DAN regions consisted of bilateral intraparietal sulcus and dorsal prefrontal cortices.

ized the BOLD response in each voxel to the voxel's average MR signal magnitude across the experimental run.

Defining DMN and DAN ROIs. We conducted an independent components analysis in FSL's Melodic (Jenkinson et al., 2012) to independently define key regions of the DMN and DAN using the resting-state data. We used an automated procedure for deciding the number of components and selected the components best resembling the DMN and DAN through visual inspection. As in earlier studies (Esterman et al., 2013, 2014; Rosenberg et al., 2015), we sought to isolate the peak 200 contiguous voxels in four regions of each network. Because some of the peak clusters had voxels with equivalent $z$-scores, the actual region sizes ranged from 200 to 203 voxels. For the DMN, we defined the precuneus, vmPFC, and bilateral lateral parietal cortices. For the DAN, we defined bilateral intraparietal sulcus and bilateral dorsal prefrontal cortex. The resulting four regions in each network are displayed in Figure 2.

Preparing the pretrial signal. To examine the relationship between spontaneous fluctuations of neural activity and modulations of attentional flexibility, we sought to identify regions of the brain for which the relationship between pretrial BOLD activity and behavioral RT differed for shift and hold trials. In particular, we were interested in determining whether a spontaneous change of activity before a cue was associated with either a corresponding increase or decrease in preparatory attentional flexibility on that trial. One metric of attentional flexibility is the magnitude of the difference in RT for attention shift versus attention hold trials (referred to here as a shift cost). By this definition, a flexible preparatory state of attentional control is associated with a smaller behavioral shift cost than is a stable preparatory state. Therefore, we applied a similar logic as Leber and colleagues (2008; see also Leber, 2010) to identify whether the magnitude of the shift cost varied reliably with fluctuations in pretrial activity in the DMN and DAN.

Given our rapid event-related experimental design, the raw BOLD data would serve as a poor indicator of spontaneous pretrial activity. Rather, task-evoked BOLD responses from preceding trials could mask any weaker fluctuations in the BOLD time course that result from spontaneous fluctuations in activity. We, therefore, used a general linear model (GLM) to partial out task-evoked variability in the BOLD response as well as nuisance variability attributable to sources of noninterest such as subject motion, respiration, and scanner artifacts. Such an 
approach is feasible because spontaneous fluctuations and task-evoked responses in brain activity are additive (Fox et al., 2006; Al-Aidroos et al., 2012). For each of the four event types of interest (hold attention left, hold attention right, shift attention from left to right, and shift attention from right to left), we modeled the BOLD response with a set of 12 finite impulse response (FIR) tent basis functions (one for each volume acquired starting at the onset of the cue and continuing to $24 \mathrm{~s}$ post-cue). As in earlier studies (Leber, 2010; Al-Aidroos et al., 2012), we sought to also remove nuisance variability from the BOLD time course. We therefore entered the six demeaned parameters of subject motion into the model and also regressed out any linear or quadratic drift in the signal. Last, we entered the BOLD time course of activity from a cluster of voxels falling inside deep white matter as well as the mean of all voxels in the wholebrain dataset (a measure of global brain activity) as two final regressors of noninterest (Fox et al., 2005). Time points for which the Euclidean norm of the motion derivative exceeded $0.3^{\circ}$, along with the preceding time point, were censored from the model as well as from all later reported fMRI analyses. Censoring resulted in a loss of $<3 \%$ of all acquired volumes. Trials in which participants made an incorrect response or failed to respond were included in the model and were not differentiated from those in which an accurate response was made. Together, these trials comprised $<12 \%$ of the total number of trials and could not have been reliably modeled separately. Due to a programming error, a small number of accurate responses were logged as failures to respond for four of the 20 participants. Because these trials lacked behavioral RTs, they are excluded from the trial-by-trial RT prediction analyses below. Nevertheless, because these participants provided sufficient data $(>140$ trials for each condition after censoring for motion outliers), we have included their trials with recorded responses in the RT correlation analyses below. All behavioral accuracy analyses are reported with these participants excluded.

To provide a measure of spontaneous pretrial brain activity, we isolated the unexplained variance in the BOLD signal after running the GLM described above. To the extent that our regressors accounted for task-evoked responses and known sources of nuisance variability, this residual time course reflected intrinsic fluctuations in brain activity. As in earlier studies (Leber et al., 2008), we focused our trial-by-trial analysis on the single-volume acquisition preceding the cue presentation. The samples of pretrial activity used in the main analyses were therefore collected $2 \mathrm{~s}$ before the cue presentation and thus cannot reflect even the earliest task-evoked response. Importantly, although the hemodynamic response takes $4-10 \mathrm{~s}$ to peak, there are immediately measurable effects after the onset of an event of interest. Therefore, any samples taken after the time of cue onset may reflect task-related processing in addition to any carryover preparatory effects. Therefore, only samples taken before the cue's onset serve as a pure measure of the relationship between intrinsic fluctuations in preparatory brain activity and moment-bymoment attentional flexibility.

Predicting trial-by-trial fluctuations in attentional flexibility. To address the main question of interest, we identified regions of the brain for which the relationship between pretrial activity and trial-by-trial RT significantly differed for shift and hold trials. First, we tested whether pretrial fluctuations of activity in the DMN and DAN covaried with the magnitude of the behavioral cost in RT associated with shifting attention. For each ROI, we computed the correlation between trial-by-trial pretrial activity and RTs separately for attention shift and attention hold trials. First, we averaged across the voxels of each ROI individually for each sample of pretrial activity and computed the correlation between these averages of pretrial activity and RT for shift trials and hold trials independently. We then ran a two-tailed paired groups $t$ test on these correlations to determine whether the magnitude of the behavioral cost associated with shifting attention relative to holding attention varied as a function of pretrial activity in either network. In a related analysis, we binned shift and hold trial RTs according to the magnitude of pretrial activity in each network and tested whether the magnitude of the shift cost was significantly different for the third of trials with the greatest pretrial activity than for the third of trials with the lowest pretrial activity. To test the temporal specificity of the window in which BOLD predicted trial-by-trial attentional flexibility, we conducted a follow-up analysis in which we repeated the DMN and DAN comparisons for data collected at time points ranging from $4 \mathrm{~s}$ before cue onset to $4 \mathrm{~s}$ after the cue onset.

As an exploratory follow-up analysis, we next used a voxelwise approach as in earlier studies of fluctuations in cognitive flexibility (Leber et al., 2008; Leber, 2010). We again computed the voxel-by-voxel correlation between pretrial activity and behavioral RT for shift and hold trials independently for each participant, resulting in two statistical parametric maps of correlations for every participant. At the group level of analysis, we then ran a two-tailed paired-groups $t$ test to identify voxels for which the correlations, averaged across participants, differed for shift and hold trials. This analysis yielded voxels in which the magnitude of shift costs, an indicator of attentional flexibility, varied based on intrinsic pretrial brain activity.

Last, we tested whether pretrial fluctuations of activity in the regions associated with attentional stability and those associated with attentional flexibility in the whole-brain analysis uniquely accounted for variance in shift trial RT. For shift trials only, we $z$-scored the samples of pretrial activity for the two sets of regions and the behavioral RTs independently for each participant and then concatenated the scores across participants. We then used a linear regression model to test whether the two sets of regions independently accounted for variability in shift trial RT.

Identifying shift-related task-evoked activity. To compare our findings with earlier studies of covert attentional control (Chiu and Yantis, 2009), we next tested for the presence of task-evoked brain activity. We used an ARMA $(1,1)$ model with restricted maximum likelihood estimation of temporal autocorrelation in the time series to estimate the evoked response for each of the four event types of interest listed above. We modeled a period of $10 \mathrm{~s}$, starting with the time of cue onset, with a series of six FIR tent basis functions, thus making no assumptions about the shape of the hemodynamic response. Furthermore, we included the demeaned six parameters of subject motion as regressors of noninterest into the model and also corrected for linear and quadratic trends in the BOLD time course. We censored TR pairs for which the Euclidean norm of the motion derivative exceeded $0.3^{\circ}$ as above.

Whole-brain multiple-comparisons correction. Due to our hypotheses that large cortical regions would be associated with spontaneous fluctuations in attentional flexibility and the possibility of weak effects inherent in a trial-by-trial analysis of such fluctuations, we adopted a liberal twotailed height threshold of $t_{(19)}=2.09, p=0.05$ for the whole-brain spontaneous fluctuations analysis. However, for the more statistically robust contrast of evoked activity when comparing the response associated with shift and hold trials, we adopted a more stringent threshold of $t_{(19)}=2.86, p=0.01$. Given these uncorrected thresholds, all wholebrain group-level results were corrected for multiple comparisons by running 10,000 Monte Carlo simulations in 3dClustSim to determine the probability of obtaining a cluster of significant activity of a certain size given the spatial smoothness of the data. Spatial smoothness was estimated from the residuals of the GLM in which we partialled out taskevoked and nuisance sources of variability. The simulation accounted for both positive and negative voxels passing the set two-tailed height threshold given a synthetic dataset with the same voxel dimensions and spatial smoothness as the acquired data. All EPI data were masked according to the registration template resampled to isotropic $2 \mathrm{~mm}$ voxels. The resulting mask yielded an extent threshold of 214 contiguous voxels (1712 $\mathrm{mm}^{3}$ ) for the spontaneous fluctuations analysis and an extent threshold of 70 contiguous voxels $\left(560 \mathrm{~mm}^{3}\right)$ for the evoked activity contrast such that the familywise rate of error was $p<0.05$ for both.

\section{Results}

\section{Behavioral results}

We first tested whether behavioral RTs and accuracies differed for attention shift trials compared with attention hold trials. A paired-groups $t$ test revealed that participants were indeed slower to make the parity judgment on shift attention trials $(\mathrm{M}=$ 998.29, $\mathrm{SD}=117.84)$ compared with hold attention trials $(\mathrm{M}=$ $884.70, \mathrm{SD}=128.06), t_{(19)}=7.64, p<0.001$ ) (Fig. 3). Furthermore, analysis of the 16 participants with valid accuracy data revealed that there were no significant differences in behavioral 


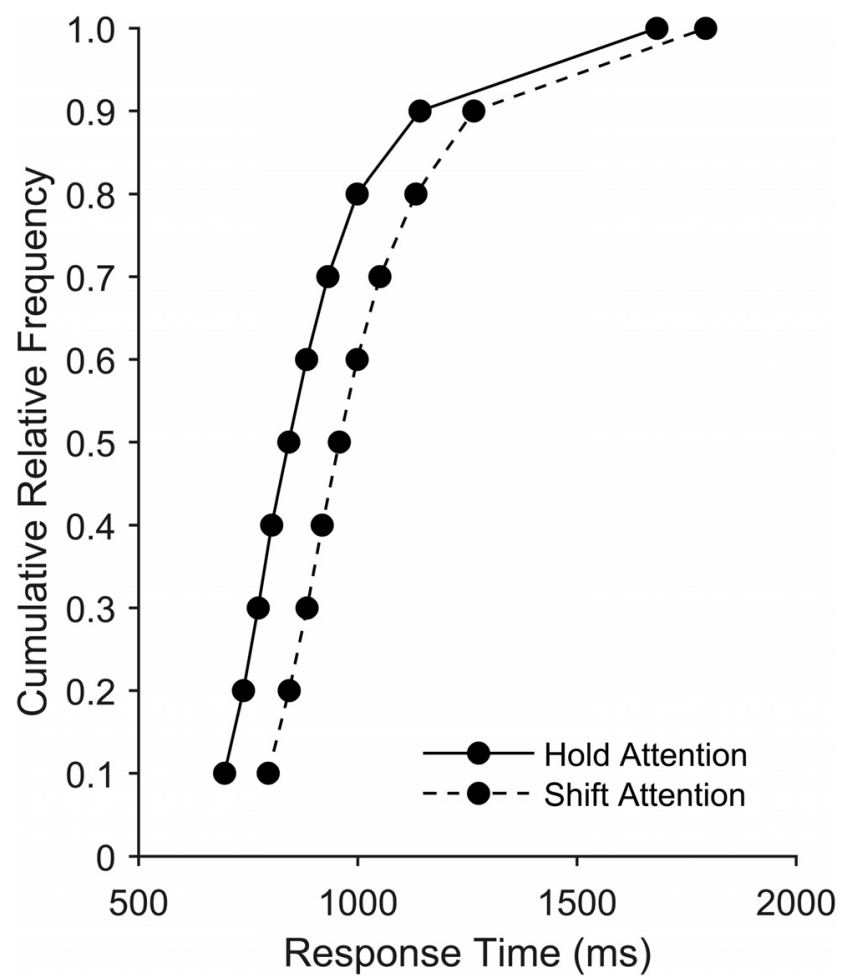

Figure 3. Vincentized plot of parity judgment response times.

Table 1. Mean behavioral RTs as a function of behavioral accuracy

\begin{tabular}{llr}
\hline & Previous trial accuracy & \\
\cline { 2 - 3 } & Accurate & Inaccurate \\
\hline Shift trials & $1014.42(104.16)$ & $1085.22(179.54)$ \\
Hold trials & $893.95(128.77)$ & $980.07(174.55)$ \\
\hline
\end{tabular}

SDs are displayed in parentheses.

accuracies for shift $(\mathrm{M}=91.49, \mathrm{SD}=6.08)$ and hold $(\mathrm{M}=91.52$, $\mathrm{SD}=7.23)$ trials $\left(t_{(15)}=0.05, p=0.965\right)$. Last, we tested whether attentional flexibility varied based on previous trial accuracy. For example, it is possible that participants adjusted attentional control settings after errors differently than after trials with an accurate response. We therefore analyzed the behavioral RT data with an additional $2 \times 2$ ANOVA with factors of current cue type (shift vs hold) and previous trial accuracy (accurate vs inaccurate). Although participants were in general slower after a trial in which they made an error $\left(F_{(1,15)}=10.64, p=0.005\right)$, there was no significant interaction of cue type and previous trial accuracy $\left(F_{(1,15)}=0.21, p=0.655\right)$ (Table 1$)$. We next investigated whether fluctuations in pretrial brain activity could predict the magnitude of the observed shift cost in RT.

\section{Imaging results}

Spontaneous fluctuations predict attentional flexibility

We tested whether pretrial activity served as an index of spatial attentional flexibility on a trial-by-trial basis. To isolate spontaneous fluctuations in the BOLD time series, we extracted samples of pretrial activity after partialling out task-evoked activity and nuisance variability. Although we chose to model behavioral error trials with correct response trials in the GLM, the RT correlation analyses included only those trials in which participants responded correctly within the 2 s response window. Because we extracted the single volume recorded two seconds before the cue onset for each trial from the residual time course of BOLD activ-
Table 2. Mean pearson correlations $(r)$ between pretrial brain activity and RT

\begin{tabular}{llr}
\hline & \multicolumn{2}{l}{ Region of interest } \\
\cline { 2 - 3 } & \multicolumn{1}{l}{ DMN } & \multicolumn{1}{c}{ DAN } \\
\hline Shift trials & $0.07^{* * *}(0.08)$ & $-0.05^{* * *}(0.05)$ \\
Hold trials & $0.00(0.09)$ & $-0.04^{* *}(0.06)$ \\
\hline
\end{tabular}

SDs are displayed in parentheses.

${ }^{* * *} p<0.001$ when testing against 0 ; ${ }^{* *} p<0.01$ when testing against 0 .

ity, our pretrial measure cannot reflect an early response to the shift or hold cue. For each participant, we computed the correlation between these samples of pretrial activity and RTs for shift and hold trials separately.

\section{$D M N$ and DAN contributions to attentional flexibility}

Given our hypothesis that both the DMN and DAN may be involved in spontaneous fluctuations in attentional flexibility, we first independently defined each network and conducted an ROI analysis (see Materials and Methods). For each participant and each network, we separately computed the correlation between pretrial activity and RT and then subjected these $r$-statistics to a group-level paired-groups $t$ test. For both networks, the distributions of shift and hold trial correlations were normal according to Kolmogorov-Smirnov tests with Lilliefors significance correction $(p>0.095)$. We therefore do not Fisher $z$-transform the correlations in the analyses reported below. However, the conclusions of all tests reported here remain identical when a Fisher transform is applied. Our analysis was designed to identify both regions in which increases of activity were associated with a decrease of shift costs, as in earlier studies (Leber et al., 2008), and regions in which increases of activity were associated with an increase of shift costs. Furthermore, fluctuations in pretrial activity could be associated with a change in shift trial RT only, hold trial RT only, or both shift and hold trial RT. Although the relationship between pretrial activity and RT for shift trials did not differ from that of hold trials for the DAN $\left(t_{(19)}=-0.63, p=\right.$ $0.539)$, attentional flexibility did covary with pretrial fluctuations of activity in the DMN $\left(t_{(19)}=3.55, p=0.002\right.$ ) (see Table 2 for mean correlations). Moreover, when averaging across participants, shift trial RTs increased as a function of DMN pretrial activity $\left(t_{(19)}=4.40, p<0.001\right)$, whereas there was no significant change in hold trial RTs $\left(t_{(19)}=0.11, p=0.918\right)$. Conversely, high pretrial activity in the DAN was associated with faster response times for both attention shift $\left(t_{(19)}=-4.40, p<0.001\right)$ and attention hold $\left(t_{(19)}=-3.36, p=0.003\right)$ trials. As a direct comparison of the relationships between pretrial activity and RT across the two networks, we followed up the above analyses by subjecting the correlations to a $2 \times 2$ ANOVA with factors of cue type (shift vs hold) and network (DMN vs DAN). In addition to significant main effects of cue type $\left(F_{(1,19)}=6.99, p=0.016\right)$ and network $\left(F_{(1,19)}=20.35, p<0.001\right)$, there was a significant interaction of the two factors $\left(F_{(1,19)}=7.87, p=0.011\right)$, providing evidence that pretrial spontaneous fluctuations of activity in the DMN and DAN carry different consequences for attentional flexibility. Critically, whereas pretrial increases of activity in the DAN were associated with a speeding of RT regardless of cue type, perhaps due to increased task engagement, only pretrial fluctuations in the DMN covaried with trial-by-trial attentional flexibility.

To better illustrate how behavior changed as a function of pretrial DMN and DAN activity, we binned shift and hold trial RTs into three groups each according to the amplitude of pretrial activity and tested whether the magnitude of the shift cost dif- 
fered in the third of trials with the lowest pretrial activity versus the third of trials with the highest pretrial activity. When binning based on DMN activity, a $2 \times 2$ repeated-measures ANOVA with factors of cue type (shift vs hold) and pretrial activity (low vs high) revealed significant main effects of both cue type $\left(F_{(1,19)}=\right.$ 47.00, $p<0.001)$ and pretrial activity $\left(F_{(1,19)}=7.69, p=0.012\right)$ such that participants were, overall, slower when DMN activity was high. Importantly, consistent with the correlation analysis above, there was a significant interaction of cue type and pretrial activity $\left(F_{(1,19)}=6.65, p=\right.$ 0.018; Fig. $4 A$ ). When we repeated the same analysis using the DAN as the ROI, we found significant main effects of cue type $\left(F_{(1,19)}=43.51, p<0.001\right)$ and pretrial activity $\left(F_{(1,19)}=30.80, p<0.001\right)$, but no significant interaction $\left(F_{(1,19)}=0.02, p=0.880\right.$; Fig. $\left.4 B\right)$. Although participants had shorter RTs when DAN pretrial activity was high than when it was low, this change in RT was equivalent for shift and hold trials and therefore does not indicate a change in the readiness to update spatial attentional selections.

In the above analyses, we collected samples of pretrial activity from the volume acquired closest in time, but preceding, the cue onset. Given our design, these samples came $2 \mathrm{~s}$ before the appearance of the cue. We focused our analysis on this particular time point because we were interested in whether intrinsically generated fluctuations occurring prior the cue's onset predicted trial-by-trial flexibility, as has been demonstrated previously in the domains of task switching and susceptibility to attentional capture (Leber et al., 2008; Leber, 2010). To better understand the temporal dynamics of how intrinsic fluctuations in brain activity relate to ongoing changes in attentional flexibility, we repeated the above analyses using samples of brain activity ranging from $4 \mathrm{~s}$ before cue onset through $4 \mathrm{~s}$ after cue onset. For the DMN, we observed the same significant difference between the shift trial correlations and hold trial correlations at $4 \mathrm{~s}$ before cue onset $\left(t_{(19)}=2.84, p=0.010\right)$ and at the time of cue onset $\left(t_{(19)}=2.15\right.$, $p=0.045$; Fig. $5 A$ ). In both cases, one-sample $t$ tests revealed that the correlations were, on average, significantly greater than $0(t>$ $3.41, p<0.004)$, whereas the hold trial correlations did not significantly differ from $0(t<0.69, p>0.502$. Furthermore, fluctuations in the DAN failed to covary with attentional flexibility at both time points $(t<0.59, p>0.568$; Fig. $5 B)$. Our above findings are therefore not specific to samples taken $2 \mathrm{~s}$ before cue onset.

We next investigated whether DMN activity continued to differentially predict shift and hold trial RT in the temporal window after cue onset. Elevated activity within the DMN has been associated with lapses of attentional engagement (Weissman et al., 2006; e.g., Christoff et al., 2009) and the relationship between brain activity and behavioral performance may therefore change according to the temporal window of interest. In support of a more nuanced relationship between DMN activity and preparatory attentional control, the correlations between brain activity and behavioral performance for shift and hold trials did not differ at the group level for samples taken at both 2 and $4 \mathrm{~s}$ after the cue presentation $(t<0.33, p>0.746)$. Interestingly, at both 2 and $4 \mathrm{~s}$ after cue onset, DMN activity was associated with increased RT for both shift and hold trials $(t>5.34, p<0.001)$. Late increases in activity were therefore associated with slow task performance
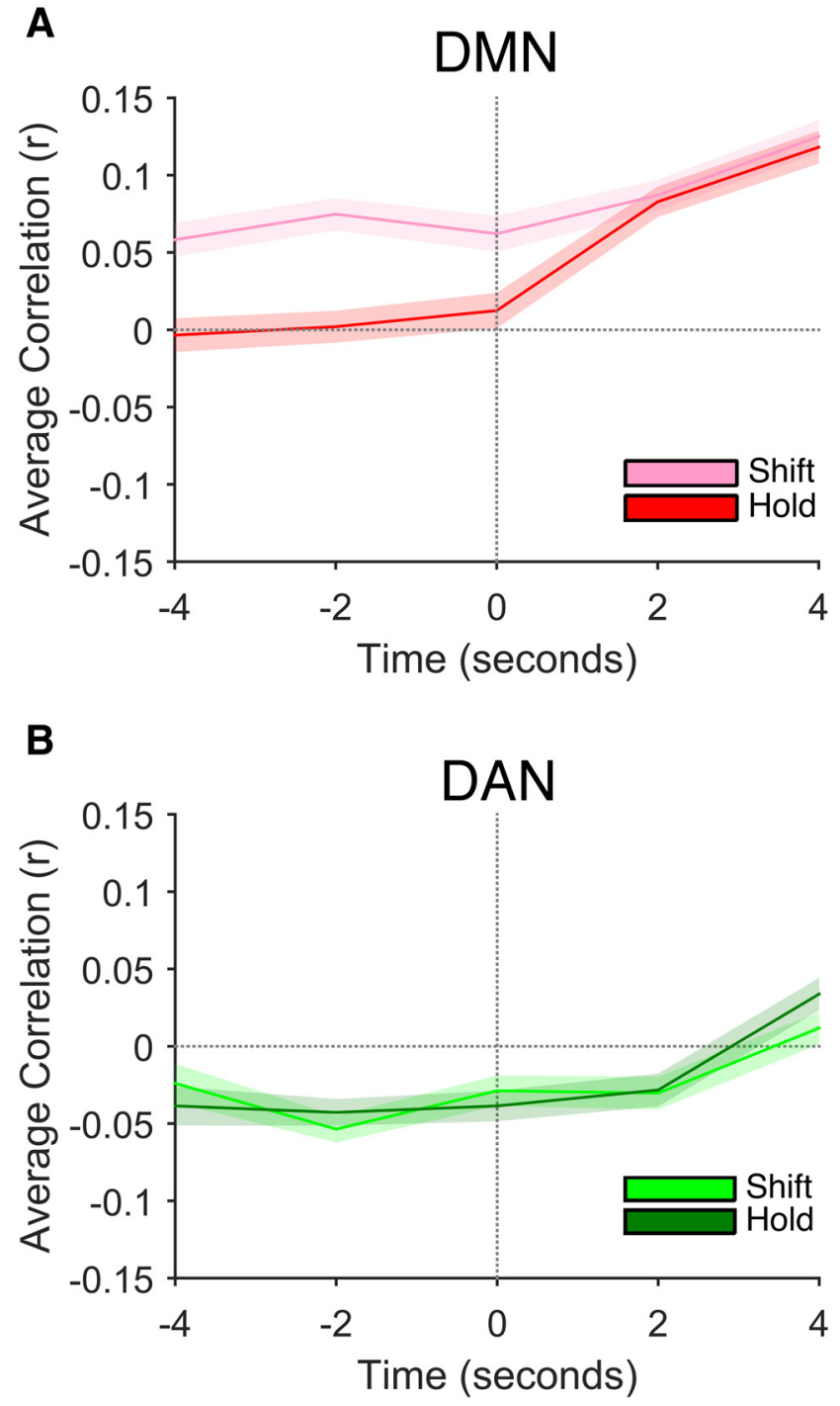

Figure 5. Mean correlations between intrinsic brain activity and behavioral RT as a function of time relative to cue onset for the $\operatorname{DMN}(\boldsymbol{A})$ and $\operatorname{DAN}(\boldsymbol{B})$. Time point 0 denotes the time of cue onset.

regardless of cue type. As in the temporal window preceding cue onset, we observed no difference in the shift and hold trial correlations for the DAN in the post cue window $(t<1.06, p>0.306)$.

Last, we compared the relationship between DMN brain activity and attentional flexibility in the temporal window preced- 


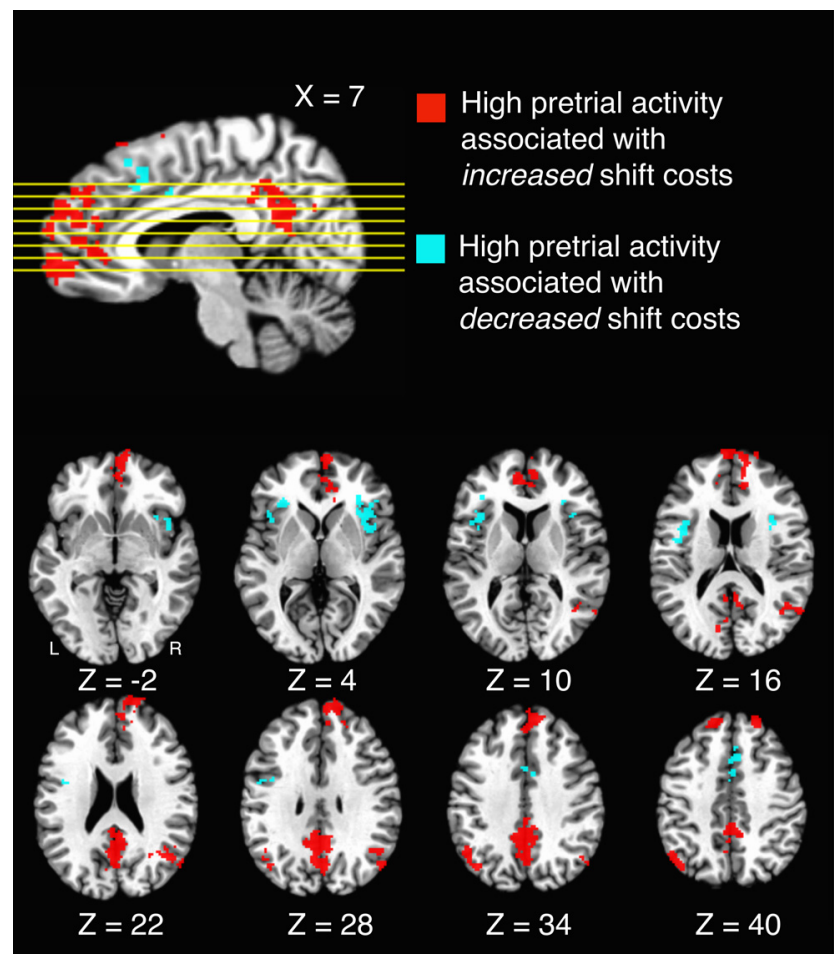

Figure 6. Areas in which intrinsic fluctuations predicted moment-by-moment attentional flexibility. High pretrial activity in areas shown in red was associated with an increase in attentional stability; high pretrial activity in areas shown in blue was associated with an increase in attentional flexibility.

ing cue onset directly with that after cue onset by collapsing the precue ( 2 and $4 \mathrm{~s}$ before the cue) and the post-cue ( 2 and $4 \mathrm{~s}$ after the cue) correlations for each participant. A repeated-measures ANOVA with factors of cue type (shift vs hold) and temporal window (pre cue vs post cue) yielded significant main effects of cue type $\left(F_{(1,19)}=6.57, p=0.019\right)$ and temporal window $\left(F_{(1,19)}\right.$ $=24.21, p<0.001$ ), which were qualified by a significant cue by window interaction $\left(F_{(1,19)}=6.01, p=0.024\right)$. Only early fluctuations in DMN activity predicted ongoing changes in preparatory states of attentional flexibility.

\section{Whole-brain analysis}

After the targeted ROI approach, we conducted a whole-brain analysis to determine whether spontaneous fluctuations of activity in any additional brain regions predicted ongoing changes in attentional flexibility. Rather than averaging voxels within an ROI, we computed the shift and hold trial correlations independently for each voxel and conducted a voxelwise paired-groups $t$ test to identify regions in which the magnitude of shift costs varied based on pretrial activity (Leber, 2010). Given our interest in relating intrinsic fluctuations of preparatory brain activity to moment-by-moment changes in behavioral performance, we focused on samples taken $2 \mathrm{~s}$ before the cue onset for all remaining analyses. As illustrated in Figure 6, we identified several regions for which pretrial fluctuations of activity predicted moment-bymoment increases or decreases in attentional flexibility, in addition to those already contained in the DMN ROI. In addition to significant clusters in the precuneus, the vmPFC, and the bilateral lateral parietal cortex, which largely overlapped with those areas tested as the DMN ROI, the left anterior superior frontal gyrus (aSFG) and right anterior medial frontal cortex were associated with states of increased attentional stability (see Table 3 for coordinates, volumes, and mean correlations). Conversely, significant clusters falling in bilateral anterior insula (AI) as well as the presupplementary motor area/supplementary motor area (preSMA/SMA) demonstrated the opposite relationship. Specifically, shift costs decreased as a function of pretrial activity in the AI and pre-SMA/SMA, suggesting that spontaneous increases of activity were associated with an increase in attentional flexibility.

Although the primary focus of the experiment was to identify regions in which the predictive relationship between pretrial activity and behavioral performance differed for shift and hold attention trials, we also tested the relative contribution of the stability- and flexibility-associated regions identified above when serving as predictors of RT together. We focused on shift attention trials because the DMN ROI analysis revealed that DMN pretrial activity did not significantly covary with hold trial RT. We averaged across the stability-associated and flexibilityassociated regions for each participant to yield two samples of pretrial activity. We then $z$-scored the samples of pretrial brain activity and behavioral RTs for each participant and concatenated across participants to run a single regression model. Last, we conducted a forward stepwise linear regression to predict RT based on pretrial activity in the stability-associated and flexibility-associated regions. In the first model, high pretrial activity within the stability associated regions was associated with an increase in shift trial RT $(\beta=0.101, t=6.19, p<0.001$, adjusted $R^{2}=0.010$ ), as would be expected from the way in which these regions were defined. Importantly, however, both stability-associated regions $(\beta=0.072, t=4.04, p<0.001)$ and flexibility-associated regions $(\beta=-0.071, t=-4.00, p<0.001)$ served as significant predictors of shift trial RT when in the regression model together (adjusted $R^{2}=0.014$ ). As reflected by the adjusted $R^{2}$ above, inclusion of both sets of regions did not yield a large boost in the percentage of variance in RT that was explained by pretrial brain activity. However, even though the predictive relationships between pretrial activity and RT are small, the important observation for the current question of interest is that they significantly differ. The multiple regression analysis supplements this finding by revealing that both sets of regions uniquely contribute to the prediction of shift trial RT.

\section{Examining task-evoked activity}

In replication of earlier studies, we also examined task-evoked activity. To determine whether group-level differences existed in evoked activity across the four trial types, we summed the $\beta$ weights of the 10 basis functions to compute the area under the curve (AUC) for each trial type. A whole-brain repeatedmeasures ANOVA of the AUCs revealed converging evidence in support of previous accounts of sustained and transient shiftrelated neural activity (Chiu and Yantis, 2009). As illustrated in Figure 7, there was a transient increase in activity for shift relative to hold trials within a series of frontal and parietal regions that previous studies have implicated in covert shifts of spatial attention (see Table 4 for Talairach coordinates and volumes).

Last, we tested whether there was a significant difference in evoked activity within any of the regions identified in the whole brain analysis above as indexing trial-by-trial attentional flexibility. Such a difference might have suggested that the relationship between pretrial activity and trial-by-trial flexibility was in part due to an incomplete removal of task-evoked activity from the BOLD time series. Contrary to this account, mean evoked activity for attention shift and attention hold trials did not significantly differ for any of the regions that predicted trial-by-trial attentional flexibility in the whole brain analysis $(t<1.88, p>0.075)$. 
Table 3. Regions predicting moment-by-moment flexibility in the whole-brain analysis

\begin{tabular}{|c|c|c|c|c|c|c|}
\hline \multirow{2}{*}{$\begin{array}{l}\text { Area } \\
\text { High activity associated with stability }\end{array}$} & \multicolumn{3}{|c|}{ Talairach coordinates $(\mathrm{mm})$} & \multirow[t]{2}{*}{ Volume $\left(\mathrm{mm}^{3}\right)$} & \multicolumn{2}{|l|}{ Correlation $r$ (SD) } \\
\hline & & & & & & \\
\hline B precuneus & - & - & - & 8,192 & $0.04(0.05)$ & $-0.01(0.04)$ \\
\hline R hemisphere & 3 & -49 & 30 & & & \\
\hline L hemisphere & -1 & -41 & 38 & & & \\
\hline R anterior medial frontal cortex & 19 & 45 & 40 & 4,776 & $0.04(0.04)$ & $-0.01(0.04)$ \\
\hline B ventromedial prefrontal cortex & - & - & - & 3,128 & $0.05(0.04)$ & $0.00(0.04)$ \\
\hline R hemisphere & 5 & 59 & -2 & & & \\
\hline L hemisphere & -9 & 41 & 12 & & & \\
\hline L anterior superior frontal gyrus & -9 & 27 & 54 & 2,976 & $0.04(0.04)$ & $-0.02(0.04)$ \\
\hline R lateral parietal cortex & 53 & -59 & 14 & 2,432 & $0.04(0.04)$ & $-0.02(0.04)$ \\
\hline L lateral parietal cortex & -47 & -61 & 38 & 1,856 & $0.05(0.06)$ & $-0.01(0.06)$ \\
\hline \multicolumn{7}{|l|}{ High activity associated with flexibility } \\
\hline Ranterior insula & 35 & 15 & 4 & 2,208 & $-0.04(0.03)$ & $0.01(0.04)$ \\
\hline L anterior insula & -39 & 5 & 16 & 1,984 & $-0.04(0.02)$ & $0.02(0.04)$ \\
\hline $\begin{array}{l}\text { B presupplementary motor area/ } \\
\text { supplementary motor area }\end{array}$ & - & - & - & 1,720 & $-0.04(0.03)$ & $0.01(0.03)$ \\
\hline R hemisphere & 7 & 17 & 44 & & & \\
\hline L hemisphere & -1 & 7 & 36 & & & \\
\hline
\end{tabular}

Talairach coordinates indicate the peak voxel within each cluster (familywise error $p<0.05$ ). The mean Pearson correlations between pretrial activity and behavioral RT are displayed separately for shift and hold attention trials for each region.

R, Right; L, left; B, bilateral cluster spanning both hemispheres (peak coordinates are displayed for each hemisphere).

\section{Shift Attention > Hold Attention}

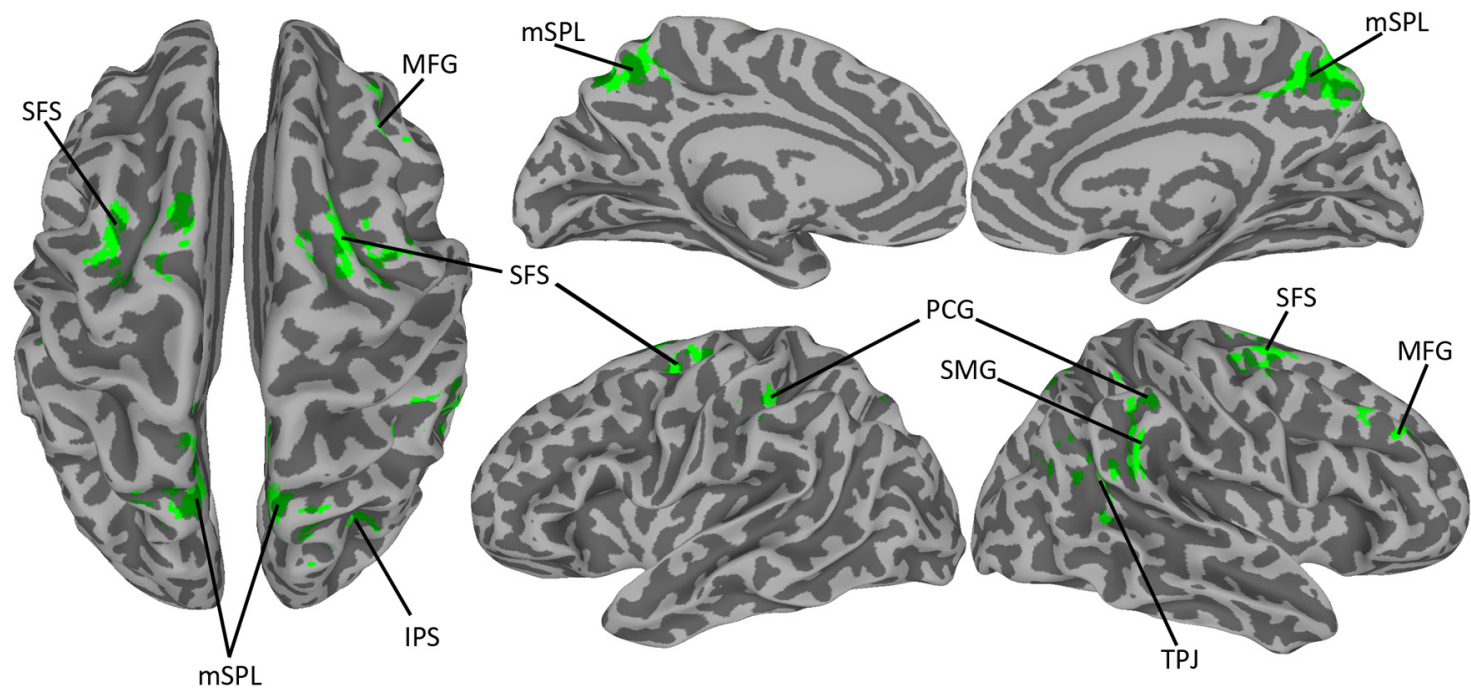

Figure 7. Transient shift-related activity on a partially inflated 3D rendering of the Talairach template used for coregistration. mSPL, Medial SPL; SFS, superior frontal sulcus; SMG, supramarginal gyrus; TPJ, temporoparietal junction; MFG, middle frontal gyrus; PCG, postcentral gyrus; IPS, intraparietal sulcus.

\section{Discussion}

In the current study, we investigated whether spontaneous fluctuations in pretrial brain activity predicted moment-bymoment changes in preparatory control over spatial attentional selection. In particular, we sought to identify regions of the brain for which the predictive relationship between pretrial activity and trial-by-trial RT differed for trials requiring a covert shift of attention compared with those in which participants held covert attention at a single peripheral location. We found that pretrial activity within the precuneus, right anterior medial frontal cortex, left aSFG, vmPFC, and bilateral lateral parietal cortex covaried with attentional flexibility such that high activity was associated with an increase in attentional shift costs. Conversely, the AI and pre-SMA/SMA demonstrated the opposite relationship such that increases of activity
Table 4. Regions demonstrating shift-related activity

\begin{tabular}{lrrrl}
\hline Area & \multicolumn{3}{l}{ Talairach coordinates $(\mathrm{mm})$} & Volume $\left(\mathrm{mm}^{3}\right)$ \\
\hline B medial superior parietal lobule & - & - & -57 & 7232 \\
$\quad$ R hemisphere & 5 & -57 & 44 & \\
$\quad$ L hemisphere & -13 & -57 & 50 & \\
R superior frontal sulcus & 25 & -9 & 58 & 2968 \\
L superior frontal sulcus & -23 & -3 & 50 & 2160 \\
R supramarginal gyrus & 59 & -45 & 28 & 1136 \\
R temporoparietal junction & 41 & -61 & 20 & 888 \\
R middle frontal gyrus & 39 & 29 & 32 & 880 \\
R postcentral gyrus & 49 & -43 & 38 & 792 \\
R intraparietal sulcus & 33 & -65 & 28 & 712 \\
L postcentral gyrus & -35 & -35 & 38 & 624 \\
\hline
\end{tabular}

Talairach coordinates indicate the peak voxel within each cluster (familywise error $p<0.05$ ).

R, Right; L, left; B, bilateral cluster spanning both hemispheres (peak coordinates are displayed for each hemisphere). 
were associated with lower shift costs, indicating a readiness to update attentional selection.

The results of the current study extend the role of key DMN regions beyond indexing generalized levels of engagement or the degree to which cognition is inwardly focused, as has been suggested previously. Instead, our findings provide evidence that ongoing spontaneous changes in activity may have differential behavioral consequences depending on whether a participant needs to shift attention or instead to maintain the focus of attention at a single location. Importantly, both shift trials and hold trials in the current experiment required participants to detect a briefly presented cue among a stream of distractor filler items and therefore placed equal demands on attentional engagement. If pretrial activity predicted pure engagement without any change in preparation to shift attention, we would have observed a similar behavioral consequence for both shift and hold trials. We found no evidence of even a trend of slower RT on hold trials being associated with higher DMN pretrial activity. Rather, it was the difference between shift and hold trials regarding the RTactivity correlation that was significant. Future research is needed to determine whether mild states of low task engagement associated with high DMN activity may predispose participants to adopt a stable attentional state that ultimately slows their time to shift attention, but not their RT to targets at the current locus of attention. Alternatively, fluctuations of pretrial activity within the precuneus, vmPFC, and lateral parietal cortex may modulate attentional stability independently of task engagement.

In contrast to samples of DMN activity taken before cue onset, those falling after the cue were associated with equivalent increases in both shift and hold trial RT. Both self-reported low task engagement during repetitive and boring sustained attention tasks (Christoff et al., 2009) and behavioral slowing in perceptual judgments requiring the focus of attention (Weissman et al., 2006) have been associated with increased DMN activity. Moreover, elevated prestimulus DMN activity has been associated with increased behavioral error rates (Eichele et al., 2008; Li et al., 2007). Our post-cue results, therefore, complement these prior findings, suggesting that high DMN activity is, under some circumstances, associated with impaired stimulus detection. However, unlike these earlier studies, only increases in DMN activity during the behavioral response window slowed performance regardless of the trial type. Importantly, although the hemodynamic response takes several seconds to peak, the response begins immediately. Our post-cue samples of activity may therefore reflect changes in brain activity associated with receiving the cue itself and with making the parity judgment. As has been demonstrated in other domains of cognition (Sadaghiani et al., 2009), the relationship between fluctuations in DMN activity and changes in behavioral performance is more nuanced than a simple change in the level of engagement. Our findings are novel in that they provide evidence that pretrial fluctuations in DMN activity carry consequences for the flexibility of attentional control beyond any changes in generalized task engagement.

Recently, others have suggested that the relationship between DMN activity and behavioral performance varies based on fluctuating cognitive states that modulate response variability (Esterman et al., 2013, 2014). In one recent study, periods of low RT variability were associated with heightened activity within the DMN and high DMN activity preceded trials in which participants made attentional lapses. Conversely, during periods of less stability, indicated by more variable RTs, DMN activity did not significantly differ before attention lapse and correct response trials. Instead, DAN activity was greater before accurate re- sponses than lapses during these less stable periods (Esterman et al., 2013). Our results are therefore convergent in suggesting that a moderate increase of activity within the DMN, but not in the executive control regions of the frontal and parietal cortices, is associated with preparatory states of behavioral stability. Importantly, our study extends these findings from a target detection paradigm to one in which participants were periodically cued to update the spatial focus of attention, enabling us to distinguish attentional stability from attentional lapses.

In addition to those regions comprising the DMN, we found evidence that the bilateral AI and the pre-SMA/SMA were also associated with trial-by-trial fluctuations in attentional flexibility, but these areas showed the opposite relationship such that participants were more ready to shift attention as pretrial activity increased. Recent advances have suggested that the AI plays a role in both saliency detection and modulating the relative engagement of different brain networks in response to a physically salient event (for review, see Menon and Uddin, 2010). Sridharan and colleagues (2008) demonstrated that the AI plays a causal role in changing the reciprocal balance of activity between the DMN and the DAN during task performance, even in the absence of a task (Sridharan et al., 2008). Although we did not find a significant relationship between modulations of attentional flexibility and components of the DAN in the current study, the inverse relationship between AI pretrial activity and the magnitude of shift costs supports the role of the AI in regulating the balance of activity between the default mode and DANs. Our findings provide additional evidence that the AI may contribute to spontaneous changes in the balance of activity across brain networks and provides an account of how these spontaneous changes relate to moment-by-moment fluctuations in behavioral performance.

One explanation for the distinction between our findings and previous research (Leber et al., 2008) may be the nature of preparing to perform a covert shift of attention versus update task set rules. In the case of task switching, a participant may be able to execute a switch of rules before the onset of the stimuli and still be able to complete the trial accurately. For example, elevated preparatory activity in regions important for carrying out a task switch may yield a behavioral advantage for task switch trials over task hold trials. However, in the domain of spatial attention, preferential selection of the to-be-attended location may prohibit detection of a visual cue at the previously attended location and consequently impair accurate performance. Intrinsic increases of activity in regions implicated in attentional orienting may therefore carry weaker behavioral advantages than in the case of task-switching. The generality of spontaneous fluctuations in cognitive flexibility across domains such as attentional selection, task set updating, and the manipulation of representations in working memory therefore remains an unanswered question.

The interaction of spontaneous changes in brain activity with other factors influencing attentional flexibility remains an important topic of inquiry. Genetic polymorphisms that regulate PFC and striatal dopamine have been associated with persistent trait-level individual differences in cognitive flexibility (Cools, 2008; Cools and D'Esposito, 2011). In particular, elevated baseline dopamine within the PFC is associated with greater cognitive stability, whereas elevated dopamine within the striatum is associated with increased flexibility at the cost of heightened distractibility (Nolan et al., 2004; Bertolino et al., 2006; Heatherton and Wagner, 2011). An area of future research, therefore, remains the relationship between the neurobiological bases of persistent individual differences in cognitive flexibility and the magnitude 
and rate of spontaneous fluctuations in preparatory control states.

The current study has important implications for understanding the mechanisms of attention and its dysfunction. For example, children with ADHD have more variable response times on tasks of response inhibition than children without histories of impairment (Vaurio et al., 2009). Prolonged periods of cognitive flexibility in situations in which there are many distractions may contribute to the inattentive symptoms that are the hallmark of ADHD. Studying the neural mechanisms behind fluctuations of attentional control may therefore improve our understanding of the neural basis of attentional flexibility and stability and how the potential dysfunction of these mechanisms may contribute to disorders of cognitive control.

We examined the neural bases of moment-by-moment fluctuations in individuals' abilities to control covert spatial attention. Our findings suggest that fluctuations of activity within components of the DMN, the AI, and the pre-SMA/SMA index fluctuations in individuals' readiness to shift or hold attention. This identification of the neural bases of spontaneous fluctuations in cognitive control will contribute to our understanding of the neural mechanisms involved in both normal variations in the control of attention and clinical impairment in psychopathology and addiction disorders.

\section{References}

Al-Aidroos N, Said CP, Turk-Browne NB (2012) Top-down attention switches coupling between low-level and high-level areas of human visual cortex. Proc Natl Acad Sci U S A 109:14675-14680. CrossRef Medline

Anderson BA, Laurent PA, Yantis S (2011b) Value driven attentional capture. Proc Natl Acad Sci U S A 108:10367-10371. CrossRef Medline

Barkley RA (1997) Behavioral inhibition, sustained attention, and executive functions: constructing a unifying theory of ADHD. Psychol Bull 121, 65-94. CrossRef Medline

Bellgrove MA, Hester R, Garavan H (2004) The functional neuroanatomical correlates of response variability: evidence from a response inhibition task. Neuropsychologia 42:1910-1916. CrossRef Medline

Bertolino A, Blasi G, Latorre V, Rubino V, Rampino A, Sinibaldi L, Caforio G, Petruzzella V, Pizzuti A, Scarabino T, Nardini M, Weinberger DR, Dallapiccola B (2006) Additive effects of genetic variation in dopamine regulating genes on working memory cortical activity in human brain. J Neurosci 26:3918-3922. CrossRef Medline

Brainard DH (1997) The psychophysics toolbox. Spat Vis 10:433-436. CrossRef Medline

Chiu YC, Yantis S (2009) A domain-independent source of cognitive control for task sets: Shifting spatial attention and switching categorization rules. J Neurosci 29:3930-3938. CrossRef Medline

Christoff K, Gordon AM, Smallwood J, Smith R, Schooler JW (2009) Experience sampling during fMRI reveals default network and executive system contributions to mind wandering. Proc Natl Acad Sci U S A 106: 8719-8724. CrossRef Medline

Cools R (2008) Role of dopamine in the motivational and cognitive control of behavior. The Neuroscientist 14:381-395. CrossRef Medline

Cools R, D'Esposito M (2011) Inverted-u-shaped dopamine actions on human working memory and cognitive control. Biol Psychiatry 69:e113e125. CrossRef Medline

Corbetta M, Shulman GL (2002) Control of goal-directed and stimulusdriven attention in the brain. Nat Rev Neurosci 3:201-215. Medline

Cox RW (1996) AFNI: software for analysis and visualization of functional magnetic resonance neuroimages. Comput Biomed Res 29:162-173. CrossRef Medline

Desimone R, Duncan J (1995) Neural mechanisms of selective visual attention. Annu Rev Neurosci 18:193-222. CrossRef Medline

Eichele T, Debener S, Calhoun VD, Specht K, Engel AK, Hugdahl K, von Cramon DY, Ullsperger M (2008) Prediction of human errors by maladaptive changes in event-related brain networks. Proc Natl Acad Sci U S A 105:6173-6178. CrossRef Medline

Esterman M, Rosenau BJ, Chiu, Y.-C., and Yantis S (2009) Decoding cogni- tive control in human parietal cortex Proc Natl Acad Sci U S A 106: 17974-17979. CrossRef

Esterman M, Noonan SK, Rosenberg M, Degutis J (2013) In the zone or zoning out? Tracking behavioral and neural fluctuations during sustained attention. Cereb Cortex 23:2712-2723. CrossRef Medline

Esterman M, Rosenberg MD, Noonan SK (2014) Intrinsic fluctuations in sustained attention and distractor processing. J Neurosci 34:1724-1730. CrossRef Medline

Fox MD, Snyder AZ, Vincent JL, Corbetta M, Van Essen DC, Raichle ME (2005) The human brain is intrinsically organized into dynamic, anticorrelated functional networks. Proc Natl Acad Sci U S A 102:9673-9678. CrossRef Medline

Fox MD, Snyder AZ, Zacks JM, Raichle ME (2006) Coherent spontaneous activity ac- counts for trial-to-trial variability in human evoked brain responses. Nat Neurosci 9:23-25. CrossRef Medline

Heatherton TF, Wagner DD (2011) Cognitive neuroscience of selfregulation failure. Trends Cogn Sci 15:132-139. CrossRef Medline

Jenkinson M, Beckmann CF, Behrens TE, Woolrich MW, Smith SM (2012) FSL. Neuroimage 62:782-790. CrossRef Medline

Leber AB (2010) Neural predictors of within-subject fluctuations in attentional control. J Neurosci 30:11458-11465. CrossRef Medline

Leber AB, Turk-Browne NB, Chun MM (2008) Neural predictors of moment-to-moment fluctuations in cognitive flexibility. Proc Natl Acad Sci U S A 105:13592-13597. CrossRef Medline

Li CS, Yan P, ${ }^{\star}$ Bergquist KL, Sinha R (2007) Greater activation of the "default" brain regions predicts stop signal errors. Neuroimage 38:640-648. CrossRef Medline

Menon V, Uddin LQ (2010) Saliency, switching, attention and control: a network model of insula function. Brain Struct Funct 214:655-667. CrossRef Medline

Nolan KA, Bilder RM, Lachman HM, Volavka J (2004) Catechol O-methylTransferase Val158 Metpolymorphism in schizophrenia: differential effects of Val and Met alleles on cognitive stability and flexibility. Am J Psychiatry 161:359-361. CrossRef Medline

Raichle ME, MacLeod AM, Snyder AZ, Powers WJ, Gusnard DA, Shulman GL (2001) A default mode of brain function. Proc Natl Acad Sci U S A 98:676-682. CrossRef Medline

Reynolds JH, Chelazzi L, Desimone R (1999) Competitive mechanisms subserve attention in macaque areas V2 and V4. J Neurosci 19:1736-1753. Medline

Rosenberg MD, Finn ES, Constable RT, Chun MM (2015) Predicting moment-tomoment attentional state. Neuroimage 114:249-256. CrossRef Medline

Sadaghiani S, Hesselmann G, Kleinschmidt A (2009) Distributed and antagonistic contributions of ongoing activity fluctuations to auditory stimulus detection. J Neurosci 29:13410-13417. CrossRef Medline

Sali AW, Anderson BA, Yantis S (2014) The role of reward prediction in the control of attention. Journal of Experimental Psychology: Human Perception and Performance 40:1654-1664. CrossRef Medline

Sali AW, Anderson BA, Yantis S (2015) Learned states of preparatory attentional control. Journal of Experimental Psychology: Learning, Memory, and Cognition 41:1790-1805. CrossRef Medline

Serences JT, Yantis S (2006) Selective visual attention and perceptual coherence. Trends Cogn Sci 10:38-45. CrossRef Medline

Sridharan D, Levitin DJ, Menon V (2008) A critical role for the right frontoinsular cortex in switching between central-executive and default-mode networks. Proc Natl Acad Sci U S A 105:12569-12574. CrossRef Medline

Vaurio RG, Simmonds DJ, Mostofsky SH (2009) Increased intra-individual reaction time variability in attention-deficity/hyperactivity disorder across response inhibition tasks with different cognitive demands. Neuropsychologia 47:2389-2396. CrossRef Medline

Volkow ND, Wang GJ, Baler RD (2011) Reward, dopamine and the control of food intake: Implications for obesity. Trends Cogn Sci 15:37-46. CrossRef Medline

Weissman DH, Roberts KC, Visscher KM, Woldorff MG (2006) The neural bases of momentary lapses in attention. Nat Neurosci 9:971-978. CrossRef Medline

Yantis S, Schwarzbach J, Serences JT, Carlson RL, Steinmetz MA, Pekar JJ, Courtney SM (2002) Transient neural activity in human parietal cortex during spatial attention shifts. Nat Neurosci 5:995-1002. CrossRef Medline 